\title{
Germinación de semillas de cuatro especies arbóreas del bosque seco tropical del Valle del Cauca, Colombia
}

\author{
Jhon Alexander Vargas-Figueroa*, Olga Lucía Duque-Palacio \& Alba Marina Torres-González \\ Grupo de Investigación "Ecología y Diversidad Vegetal", Departamento de Biología, Facultad de Ciencias Naturales \\ y Exactas, Universidad del Valle, Cali, Colombia; lexvafi13@gmail.com, olgadu64@yahoo.es, \\ alba.torres@correounivalle.edu.co \\ * Correspondencia
}

Recibido 03-IV-2014. Corregido 01-VIII-2014. Aceptado 03-IX-2014.

\begin{abstract}
Seed germination of four tree species from the tropical dry forest of Valle del Cauca, Colombia. The ecological restoration strategies for highly threatened ecosystems such as the tropical dry forest, depend on the knowledge of limiting factors of biological processes for the different species. Some of these include aspects such as germination and seed longevity of typical species present in those forests. In this study, we evaluated the effect of light and temperature on seed germination of two Fabaceae (Samanea saman and Jacaranda caucana) and two Bignoniaceae (Pithecellobium dulce and Tabebuia rosea) species having potential use in restoration, and we analyzed the seed storage behavior of these species for a three months period. To study the light effect, four levels of light quality on seeds were used (photoperiod of 12 hours of white light, darkness and light enriched in red and far-red, both for an hour each day), and we combined them with three levels of alternated temperatures $\left(20 / 25,20 / 30\right.$ and $\left.25 / 30^{\circ} \mathrm{C}-16 / 8 \mathrm{~h}\right)$. For the storage behavior, two levels of seed moisture content particular for each species were used (low: 3.5-6.1\% and high: 8.3-13.8\%), with three storage temperatures (20, 5 and $-20^{\circ} \mathrm{C}$ ) and two storage times (one and three months). The criterion for germination was radicle emergence which was measured in four replicates per treatment, and was expressed as percentage of germination (PG). There were significant differences in germination of Samanea saman and Jacaranda caucana among light and temperature treatments, with the lowest value in darkness treatments, whereas germination of Pithecellobium dulce and Tabebuia rosea did not differ between treatments (PG $>90 \%$ ). The most suitable temperature regime to promote germination in all species was $25 / 30^{\circ} \mathrm{C}$. These four species showed an orthodox seed storage behavior. We concluded that seeds of $P$. dulce, J. caucana and $T$. rosea did not have an apparent influence of all light conditions tested in their germination response, which might confer advantages in colonization and establishment processes, while $S$. saman did not germinate well in darkness. We suggest the use of seeds of $P$. dulce, $J$. caucana and $T$. rosea in ecological restoration processes, due to their tolerance and germination under a wide range of temperature and light conditions. Futhermore, seeds of $S$. saman might be used in open areas such as forest gaps. Rev. Biol. Trop. 63 (1): 249-261. Epub 2015 March 01.
\end{abstract}

Key words: Samanea saman, Pithecellobium dulce, Jacaranda caucana, Tabebuia rosea, light response, seed storage behaviour.

Las áreas urbanas y suburbanas de los valles interandinos de Colombia pertenecen a la zona de vida bosque seco Tropical (Espinal \& Montenegro, 1963). En esta zona, las familias más ricas en especies son Fabaceae y Bignoniaceae (Gentry, 1995). En cada familia, se tienen como especies representantes, entre otras, a Samanea saman (Jacq.) Merr. y Pithecellobium dulce (Roxb.) Benth. en Fabaceae (Pérez-Arbeláez, 1956; Díaz-Martín, 2005), y a Jacaranda caucana Pittier y Tabebuia rosea (Bertol) Bertero ex A. DC. en Bignoniaceae (Gentry, 2009). S. saman, P. dulce y T. rosea se consideran especies de los estadios tempranos en la sucesión vegetal de estos bosques e, incluso, se han descrito como especies 
potencialmente invasoras en sitios por fuera de su distribución natural (Space \& Flynn, 2001; Space \& Flynn, 2002; Socolowski \& Takaki, 2007; Space, Lorence, \& LaRosa, 2009; Tan, Thu, \& Dell, 2012). Sin embargo, se sabe muy poco sobre la germinación y propagación de las especies de los bosques secos tropicales, por lo cual es necesario ampliar el número de especies estudiadas para conocer las bases para una adecuada restauración de la diversidad de dichos bosques.

Los bosques secos tropicales de América Latina han sufrido una alta pérdida de área en las últimas décadas (Janzen, 1988; Corporación Autónoma Regional del Valle del Cauca, 1990; Murphy \& Lugo, 1995; Portillo-Quintero \& Sánchez-Azofeifa, 2010) y, por consiguiente, presentan actualmente un alto grado de amenaza. Estudios de línea base de la vegetación propia de bosques secos y sus dinámicas evidencian que la conservación permite el avance de las sucesiones tempranas y su recuperación (Torres et al., 2012). Sin embargo, los planes de conservación y restauración ecológica deben ser específicos para los bosques secos, debido a sus características considerablemente diferentes entre sí, y respecto a otros ecosistemas más estudiados (Vieira \& Scariot, 2006). Uno de los puntos prioritarios, es conocer la biología de las especies propias de esta zona de vida. El conocimiento de sus características ecológicas permitirá utilizar especies apropiadas para dichos planes. Para lograr un proceso económico y rápido, es indispensable el conocimiento puntual de los factores limitantes de la germinación de las especies a usar, con el fin de crear planes de propagación y restauración adecuados y lograr una eficiencia satisfactoria en la implementación de los mismos (Bradshaw, 1987; Quesada et al., 2009).

La exposición de las semillas a variaciones tanto en la calidad de la luz incidente como en la temperatura permite en ciertos casos promover la germinación de las mismas. El cambio en la luminosidad del medio puede ejercer un control positivo o negativo en la germinación, lo que en últimas funciona como un factor determinante del establecimiento y sobrevivencia de las plántulas. Esto depende en gran parte del genotipo y de las condiciones medioambientales durante la maduración de las semillas (Pons, 2000). En sitios donde la temperatura tiene variaciones marcadas, la respuesta de las semillas depende de la presencia de al menos niveles bajos de fitocromo en su estado activo, $\mathrm{P}_{\mathrm{fr}}$, y dicha respuesta está en función del número y de la amplitud de estas variaciones (Probert, 2000). Las interacciones entre la luz y la temperatura pueden variar significativamente entre especies, ya que en ciertos escenarios, la luz puede reemplazar por completo los efectos dados por alternancia de temperaturas, mientras que en otros, el efecto de la luz se reduce solamente a disminuir la amplitud necesaria en los cambios de temperatura para que la germinación se lleve a cabo (Fenner \& Thompson, 2005).

Por otro lado, el mantenimiento de la longevidad de las semillas en bancos in situ o ex situ depende de las condiciones ambientales y de la fisiología de las mismas en el almacenamiento (Harrington, 1972). La temperatura juega un papel clave en dichos procesos, ya que ésta afecta el deterioro, la latencia y el vigor de las mismas (Probert, 2000). La mayoría de las semillas tienen un comportamiento ortodoxo (Hong \& Ellis, 1996), lo que significa que pueden secarse a contenidos bajos de humedad, sin dañarse y mantener una longevidad alta en condiciones variadas (Roberts, 1973). Sin embargo, el comportamiento recalcitrante es menos frecuente y corresponde a semillas que no sobreviven a la deshidratación (Roberts, 1973), y germinan tan pronto llegan al suelo luego de ser dispersadas (Thompson, 2000). Finalmente, otras semillas tienen un comportamiento intermedio, y no sobreviven a temperaturas bajas cuando son deshidratadas (Ellis, Hong, \& Roberts, 1990; Ellis, Hong, \& Roberts, 1991).

El conocimiento sobre la respuesta germinativa de las semillas a la luz y a la temperatura, y sobre el comportamiento de las semillas en el almacenamiento, es clave para hacer conservación apropiada de las especies y entender las dinámicas de germinación que puedan ser tenidas en cuenta en procesos de 
restauración ecológica. En este estudio se evaluó el efecto de la luz y la temperatura en la germinación de semillas de S. saman, $P$. dulce, $J$. caucana y T. rosea, y se determinó el comportamiento de las semillas de estas especies en el almacenamiento.

\section{MATERIALES Y MÉTODOS}

Recolección de semillas: Las semillas de $S$. saman y $T$. rosea se recolectaron de tres y doce individuos respectivamente, ubicados en el campus de la Universidad del Valle-sede Meléndez, Cali, Colombia $\left(3^{\circ} 22 \mathrm{~N}-76^{\circ} 31 \mathrm{~W}\right)$, entre 970 y 990 m.s.n.m. Se tomó una muestra de herbario para cada especie, la cual se depositó en el Herbario CUVC ( $S$. saman: número de colección 30, O. L. Duque-Palacio; T. rosea: número de colección 228, J. A. Vargas-Figueroa). Debido a la poca producción de semillas de los individuos de $P$. dulce y $J$. caucana en el campus, éstas se compraron al proveedor de semillas forestales "El Semillero", cosechadas en cultivos ubicados en Tesalia, Huila, Colombia. Las semillas se procesaron y almacenaron en el Laboratorio de Semillas de la Universidad del Valle. Se estableció el contenido de humedad de los lotes de semillas, con la prueba de humedad a alta temperatura estandarizada para cada especie de acuerdo con las reglas del International Seed Testing Association (1999) (i.e. 10 repeticiones de $1 \mathrm{~g}$ cada una, a $130^{\circ} \mathrm{C}$ por una hora en horno de secado Tomas Scientific TSOV2G).

Evaluación del efecto de la luz y la temperatura en la germinación: Se realizó un experimento factorial de $3 \times 4$ para un total de 12 tratamientos, que consistió en (1) tres temperaturas alternadas $\left(20 / 25^{\circ} \mathrm{C}-16 / 8 \mathrm{~h}\right.$, $20 / 30^{\circ} \mathrm{C}-16 / 8 \mathrm{~h}$ y $25 / 30^{\circ} \mathrm{C}-16 / 8 \mathrm{~h}$ ), basadas en las temperaturas media, mínima y máxima para Cali, Colombia (Instituto de Hidrología, Meteorología y Estudios Ambientales, 2005), y (2) cuatro calidades de luz (fotoperiodo de 12 horas de luz blanca, oscuridad total, un pulso de luz de una hora con alta relación rojo:rojo lejano (R), y un pulso de luz de una hora con baja relación rojo:rojo lejano (RL)). Los pulsos de luz R y RL se aplicaron una sola vez a las semillas en cada tratamiento, antes de su ingreso en las incubadoras (Escobar \& Torres, 2013). Para el fotoperiodo y la alternancia de temperaturas se utilizaron incubadoras DiEs (K115U y KU115F). Para obtener oscuridad total se utilizaron bolsas negras de polietileno de alta densidad (PEAD). Para simular la luz roja $(640-670 \mathrm{~nm}$, pico en $660 \mathrm{~nm})$ se utilizaron dos tubos fluorescentes de luz blanca de 20w (Sylvania F20T12/D) con irradianza de $100.1 \mu \mathrm{mol} / \mathrm{m}^{2}$.s y R:RL $=0.00102$; para simular la luz roja lejana (690-748nm, pico en $730 \mathrm{~nm})$ se utilizaron dos capas de Filtro Lee 26 y una capa de Filtro Lee 120, los cuales filtraron la luz producida por dos bombillos incandescentes de 60w (Sylvania E-27) con irradianza de $3.31 \mu \mathrm{mol} / \mathrm{m}^{2}$.s y $\mathrm{R}: \mathrm{RL}=5.40045$ (Benvenuti, Macchia, \& Miele, 2001; Escobar \& Torres, 2013). Los tratamientos de luz R y RL se realizaron en una cámara oscura de madera.

Las pruebas de germinación se realizaron en cajas de Petri con papel absorbente, con cuatro repeticiones de 25 semillas por tratamiento para $P$. dulce, J. caucana y $T$. rosea, y cuatro repeticiones de 20 semillas por tratamiento para $S$. saman. Debido a que el fitocromo es insensible a la luz cuando las semillas están deshidratadas (McCullough \& Shropshire, 1970; Kendrick \& Spruit, 1977), se utilizaron semillas embebidas en agua desionizada por una hora. Las semillas de $S$. saman y $P$. dulce se escarificaron con un cortauñas para romper la latencia física, observada en pruebas preliminares de germinación (datos no publicados). Las semillas del tratamiento con fotoperiodo se embebieron en presencia de luz y se registró el número de semillas germinadas diariamente (emergencia de radícula $>1 \mathrm{~mm}$ ). En los demás tratamientos, las semillas se embebieron en oscuridad y se mantuvieron en esta condición antes y después del estímulo de luz y sólo se registró el número de semillas germinadas al final de la prueba. Las semillas que no germinaron en los tratamientos con oscuridad ( $\mathrm{R}$ y $\mathrm{RL}$ ), se colocaron en fotoperiodo de 12 horas de luz blanca para determinar su viabilidad. Para los datos de germinación con fotoperiodo 
se calculó el porcentaje de germinación (PG), la velocidad o tasa media de germinación $(\bar{v})$, con base en el coeficiente de velocidad de Nichols \& Heydecker (1968), el tiempo medio de germinación $(\bar{t})$ (Ranal \& Santana, 2006) y los índices de sincronización de Primack (1980) (Z) y de Labouriau \& Valadares (1976) $(\bar{E})$. Para los datos de germinación en oscuridad (R y RL) se calculó sólo el PG.

Determinación del comportamiento en el almacenamiento: Se siguió el protocolo descrito por Hong \& Ellis (1996). Se realizó un experimento factorial de $3 \times 2 \times 2$ para un total de 12 tratamientos, que consistió en (1) tres temperaturas de almacenamiento $(20,5 \mathrm{y}$ $-20^{\circ} \mathrm{C}$ ), (2) dos contenidos de humedad de la semilla (S. saman, 11.5 y $6.1 \%$; P. dulce, 13.8 y $5.5 \%$; J. caucana, 8.4 y $3.5 \%$; T. rosea, 8.3 y $3.6 \%)$, y (3) dos tiempos de almacenamiento (1 y 3 meses). Las semillas procesadas se empacaron al vacío en bolsas de aluminio con una selladora Fuji Impulse V-300, en grupos de 80 a 100 semillas para cada tratamiento. Para el almacenamiento a $-20^{\circ} \mathrm{C}$ se usó un congelador industrial Indufrial $\mathrm{ICH}-11$, para $5^{\circ} \mathrm{C}$ se usó una nevera con escarcha Haceb Superstar y para $20^{\circ} \mathrm{C}$ se utilizó un cuarto climatizado. Para determinar la viabilidad de las semillas en cada tratamiento de almacenamiento, se realizaron pruebas de germinación inmediatamente después de terminar cada tiempo de almacenamiento, en cajas de Petri con papel absorbente, a una temperatura alternada de $25 / 30^{\circ} \mathrm{C}$, con cuatro repeticiones de 25 semillas para $P$. dulce, J. caucana y T. rosea, y cuatro repeticiones de 20 semillas para $S$. saman. Se registró el número de semillas germinadas al final de la prueba (emergencia de radícula $>1 \mathrm{~mm}$ ), y se calculó el PG total para cada tratamiento de cada especie.

Los datos fueron analizados con el programa Minitab® 16.1.0 Statistical Software de Minitab Inc. Los porcentajes de germinación (PG) se transformaron con la función arcoseno, de acuerdo con Zar (1996). Se realizaron ANOVAs de dos factores para cada especie y pruebas de Tukey posteriores para comparar los tratamientos de luz y temperatura de cada una, con un nivel de significancia del $95 \%$ $(\alpha=0.05)$. Adicionalmente, se usó una prueba de Kruskal-Wallis para determinar la existencia de diferencias significativas en los valores de velocidad, tiempo e índices de sincronización obtenidos en cada especie en las temperaturas alternadas, con el mismo nivel de significancia, y se realizaron comparaciones múltiples de las medias entre cada variable y las temperaturas, de acuerdo con Siegel y Castellan (1988), para determinar las diferencias significativas puntuales.

\section{RESULTADOS}

Efecto de la luz y la temperatura en la germinación: Se registró germinación en todos los tratamientos de luz y temperatura evaluados. Los porcentajes de germinación (PG) en las pruebas de oscuridad, rojo y rojo lejano para $S$. saman y $J$. caucana presentaron diferencias significativas en varios tratamientos en cada especie, en comparación con los $\mathrm{PG}$ registrados en $P$. dulce y T. rosea, los cuales fueron mayores al $90 \%$ y similares entre los tratamientos para cada especie.

En S. saman, hubo interacción entre los dos factores evaluados $(p=0.0040)$. El PG fue mayor en el tratamiento con fotoperiodo de 12 horas de luz combinado con una temperatura alternada de $20 / 30^{\circ} \mathrm{C}$ (Fig. 1a). Este valor fue significativamente mayor respecto a los demás tratamientos (Tukey, $0.0354 \geq \mathrm{p} \geq 0.0001$ ), excepto para el $\mathrm{PG}$ en temperatura $25 / 30^{\circ} \mathrm{C}$ con fotoperiodo de 12 horas de luz y con RL (Tukey, $\mathrm{p}=0.0611$ ). Los valores más bajos de PG se registraron en los tratamientos con oscuridad, con un $\mathrm{PG}$ menor en $25 / 30^{\circ} \mathrm{C}$. Este valor fue significativamente diferente respecto a los valores de PG para los tres tratamientos con fotoperiodo de 12 horas de luz (Tukey, $0.0133 \geq \mathrm{p} \geq 0.0001$ ), y para los tratamientos en $20 / 25^{\circ} \mathrm{C}$ y $25 / 30^{\circ} \mathrm{C}$ con RL (Tukey, $\mathrm{p}=0.0135$ y 0.0038 , respectivamente). En contraste, en $P$. dulce no hubo interacción entre los factores $(\mathrm{p}=0.3982)$. Los valores de PG registrados fueron mayores al $90 \%$ en todos los tratamientos 

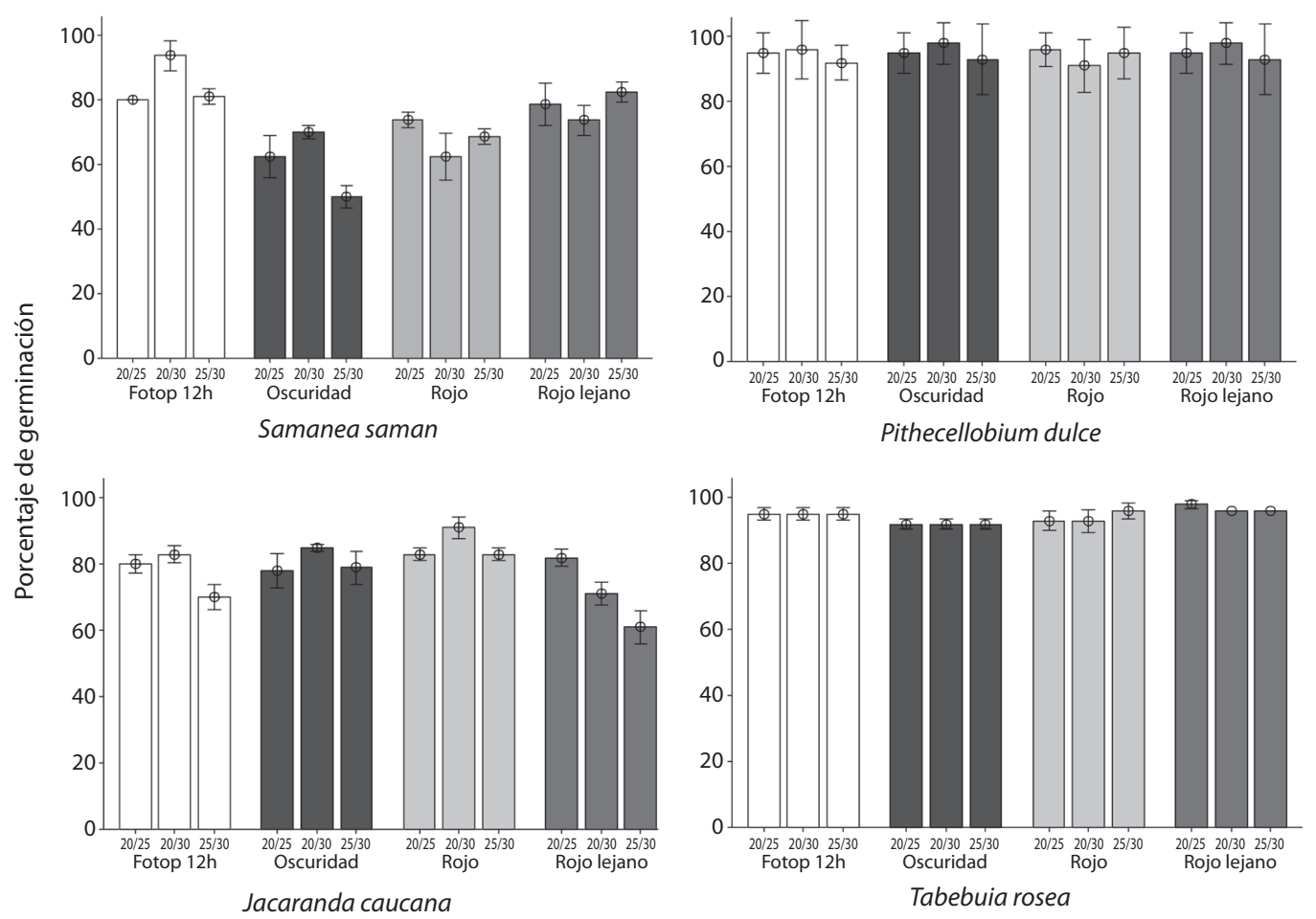

Fig. 1. Porcentaje de germinación de semillas de dos especies de Fabaceae y dos de Bignoniaceae, sometidas a cuatro calidades de luz y tres temperaturas alternadas. Las líneas verticales representan el error estándar de la media.

Fig. 1. Seed germination percentage of two Fabaceae and two Bignoniacea species, tested for four light qualities and three alternate temperatures. Vertical lines are the standard error of the mean.

(Fig. 1b), sin diferencias significativas entre ellos (Tukey, $1.0000 \geq \mathrm{p} \geq 0.505$ ). La viabilidad de las semillas de $S$. saman y $P$. dulce no germinadas en los tratamientos de oscuridad, $\mathrm{R}$ y RL fue del $0 \%$.

En J. caucana, tampoco hubo interacción entre los factores calidad de luz y temperatura alternada $(\mathrm{p}=0.0572)$. Los valores de PG estuvieron entre el 60 y el $90 \%$. El mayor valor se obtuvo en la temperatura $20 / 30^{\circ} \mathrm{C}$ con R y los menores valores en $25 / 30^{\circ} \mathrm{C}$ con RL (Fig. 1c). El PG en la temperatura $20 / 30^{\circ} \mathrm{C}$ con R fue significativamente diferente respecto al registrado en la temperatura de $25 / 30^{\circ} \mathrm{C}$ con fotoperiodo de 12 horas de luz (Tukey, $\mathrm{p}=0.0020$ ) y a los PG registrados en $20 / 30^{\circ} \mathrm{C}$ y $25 / 30^{\circ} \mathrm{C}$ con $\mathrm{RL}$ (Tukey, $\mathrm{p}=0.0031$ y 0.0001 respectivamente). El valor de PG más bajo, registrado en $25 / 30^{\circ} \mathrm{C}$ con RL, fue significativamente diferente en comparación con los valores en la temperatura de $20 / 30^{\circ} \mathrm{C}$ con fotoperiodo de 12 horas de luz y con oscuridad (Tukey, $\mathrm{p}=0.0259$ y 0.0096 respectivamente), en la temperatura $20 / 25^{\circ} \mathrm{C}$ con RL (Tukey, $\mathrm{p}=0.0422$ ) y con todos los tratamientos con $\mathrm{R}$ (Tukey, $0.0268 \geq \mathrm{p} \geq 0.0001$ ). En $T$. rosea, igualmente no se obtuvo interacción entre los factores evaluados $(\mathrm{p}=0.8187)$. Incluso, los valores de PG registrados fueron mayores al $90 \%$ en todos los tratamientos (Fig. 1d), sin diferencias significativas entre ellos (Tukey, $1.0000 \geq \mathrm{p} \geq 0.8159$ ). La viabilidad de las semillas de $J$. caucana y de $T$. rosea no germinadas en los tratamientos de oscuridad, $\mathrm{R}$ y RL fue del $0 \%$.

Por otro lado, las semillas de $S$. saman y $P$. dulce presentaron un tiempo medio de germinación y una velocidad media de germinación similares en las tres temperaturas alternadas 
para cada especie (Kruskal-Wallis, S.saman: $\mathrm{p}=0.639$ para ambas variables; $P$. dulce: $\mathrm{p}=0.276$ para ambas variables) (Cuadro 1 ). $\mathrm{La}$ sincronización en el proceso de germinación no difirió significativamente en las dos especies (Kruskal-Wallis, S. saman: $\mathrm{p}=0.668$ para $Z$ y $\mathrm{p}=0.219$ para $\bar{E} ; P$. dulce: $\mathrm{p}=0.437$ para $Z$ y $\mathrm{p}=0.276$ para $\bar{E}$ ). Las semillas de $J$. caucana y T. rosea presentaron un tiempo medio de germinación significativamente menor con la temperatura $25 / 30^{\circ} \mathrm{C}$ en comparación con el tiempo registrado en $20 / 25^{\circ} \mathrm{C}$, y, por consiguiente, una velocidad media de germinación significativamente mayor entre estos tratamientos (KruskalWallis, $\mathrm{p}=0.005$ en ambas especies para las dos variables) (Cuadro 1). La sincronización de este proceso en ambas especies fue baja. Además, en $T$. rosea no hubo diferencias significativas entre las tres temperaturas alternadas para los dos índices calculados (Kruskal-Wallis, $1.000 \geq \mathrm{p} \geq 0.092$ ), mientras que en $J$. caucana fue significativamente menor en $20 / 25^{\circ} \mathrm{C}$ para el índice $Z$ (Kruskal-Wallis, $\mathrm{p}=0.009$ ).
Viabilidad de semillas luego del almacenamiento: En $S$. saman y $P$. dulce, las semillas mantuvieron un alto porcentaje de germinación después de uno y tres meses de conservación ex situ, en todos los contenidos de humedad probados, sin diferencias significativas entre los tratamientos (Tukey, S. saman: $1.0000 \geq \mathrm{p} \geq 0.6455$; P. dulce: $1.0000 \geq \mathrm{p} \geq 0.9793$ ), (Figs. 2a, 2b, 2c y 2 d). De manera similar, en $J$. caucana y $T$. rosea, las semillas conservaron la viabilidad en las dos condiciones de deshidratación probadas, después de estar almacenadas durante el mismo tiempo, sin registrarse diferencias significativas (Tukey, J. caucana: $1.0000 \geq \mathrm{p} \geq 0.4713$; T. rosea: $1.0000 \geq \mathrm{p} \geq 0.1270$ ), (Figs. $2 \mathrm{e}, 2 \mathrm{f}, 2 \mathrm{~g}$ y $2 \mathrm{~h}$ ). Por lo tanto, las semillas de las cuatro especies tuvieron un comportamiento ortodoxo en el almacenamiento.

\section{DISCUSIÓN}

Entre las especies de Fabaceae, la germinación en $S$. saman presentó una variación significativa entre los diferentes tratamientos,

CUADRO 1

Variables fisiológicas de la germinación de dos especies de Fabaceae y dos de Bignoniaceae, sometidas a fotoperiodo de 12 horas de luz con tres temperaturas alternadas (Promedio \pm DE)

TABLE 1

Physiological variables of seed germination of two Fabaceae and two Bignoniaceae species, tested for photoperiod of 12 hours in white light and three alternate temperatures (Mean+SD)

\begin{tabular}{lccccc} 
Especie & $\begin{array}{c}\text { Temperatura } \\
\text { alternada }\left({ }^{\circ} \mathrm{C}\right)\end{array}$ & $\begin{array}{c}\text { Tiempo medio de } \\
\text { germinación }(\bar{t}, \text { día })\end{array}$ & $\begin{array}{c}\text { Velocidad media de } \\
\text { germinación }(\bar{v}, \text { semilla/día })\end{array}$ & $\begin{array}{c}\text { Índice de sincronización } \\
\text { de Primack }(Z)\end{array}$ & $\begin{array}{c}\text { Índice de sincronización de } \\
\text { Labouriau \& Valadares }(\bar{E} \text {, bit })\end{array}$ \\
S. saman & $20 / 25$ & $2.25 \pm 0.23 \mathrm{a}$ & $0.45 \pm 0.05 \mathrm{a}$ & $0.34 \pm 0.12 \mathrm{a}$ & $1.59 \pm 0.34 \mathrm{a}$ \\
& $20 / 30$ & $2.31 \pm 0.09 \mathrm{a}$ & $0.43 \pm 0.02 \mathrm{a}$ & $0.33 \pm 0.05 \mathrm{a}$ & $1.20 \pm 0.30 \mathrm{a}$ \\
P. dulce & $25 / 30$ & $2.12 \pm 0.28 \mathrm{a}$ & $0.48 \pm 0.06 \mathrm{a}$ & $0.31 \pm 0.17 \mathrm{a}$ & $1.20 \pm 0.47 \mathrm{a}$ \\
& $20 / 25$ & $2.10 \pm 0.09 \mathrm{a}$ & $0.48 \pm 0.02 \mathrm{a}$ & $0.78 \pm 0.09 \mathrm{a}$ & $0.38 \pm 0.33 \mathrm{a}$ \\
& $20 / 30$ & $2.03 \pm 0.04 \mathrm{a}$ & $0.49 \pm 0.01 \mathrm{a}$ & $0.87 \pm 0.16 \mathrm{a}$ & $0.17 \pm 0.21 \mathrm{a}$ \\
J. caucana & $25 / 30$ & $2.02 \pm 0.04 \mathrm{a}$ & $0.49 \pm 0.01 \mathrm{a}$ & $0.83 \pm 0.07 \mathrm{a}$ & $0.06 \pm 0.13 \mathrm{a}$ \\
& $20 / 25$ & $12.22 \pm 0.88 \mathrm{a}$ & $0.08 \pm 0.01 \mathrm{a}$ & $0.16 \pm 0.01 \mathrm{a}$ & $2.02 \pm 0.23 \mathrm{a}$ \\
T. rosea & $20 / 30$ & $9.97 \pm 0.69 \mathrm{ab}$ & $0.10 \pm 0.01 \mathrm{ab}$ & $0.36 \pm 0.16 \mathrm{~b}$ & $1.28 \pm 0.54 \mathrm{a}$ \\
& $25 / 30$ & $7.75 \pm 0.59 \mathrm{~b}$ & $0.13 \pm 0.01 \mathrm{~b}$ & $0.22 \pm 0.04 \mathrm{ab}$ & $1.19 \pm 0.29 \mathrm{a}$ \\
& $20 / 25$ & $6.48 \pm 0.33 \mathrm{a}$ & $0.15 \pm 0.01 \mathrm{a}$ & $0.35 \pm 0.04 \mathrm{a}$ & $1.64 \pm 0.08 \mathrm{a}$ \\
& $20 / 30$ & $5.37 \pm 0.18 \mathrm{ab}$ & $0.19 \pm 0.01 \mathrm{ab}$ & $0.41 \pm 0.07 \mathrm{a}$ & $1.49 \pm 0.28 \mathrm{a}$ \\
\hline
\end{tabular}

Los valores con letras diferentes indican diferencias significativas $(\alpha=0.05)$.

Values with different letters indicate significance differences $(\alpha=0.05)$. 
Samanea saman C.H. 11.5\%

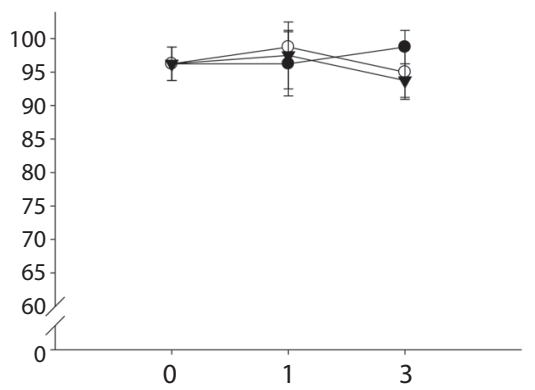

Pithecellobium dulce C.H. 13.8\%

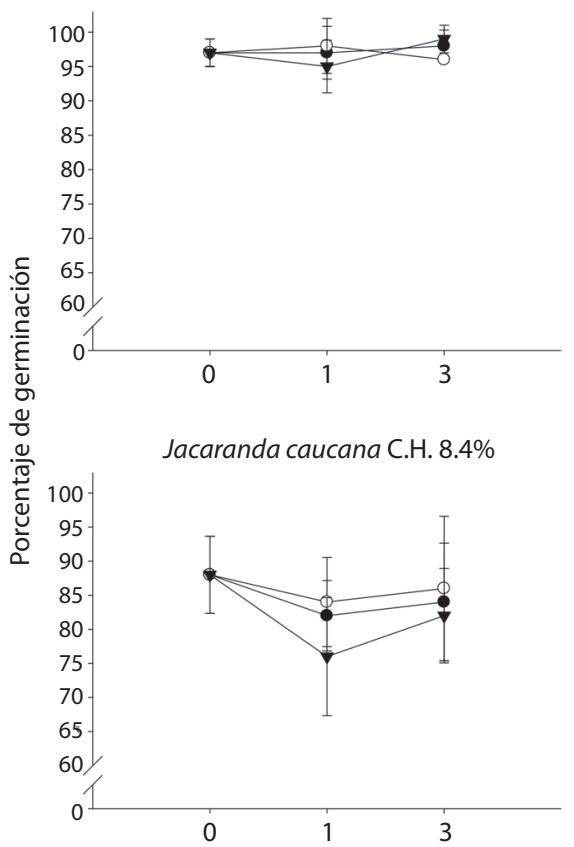

Tabebuia rosea C.H. 8.3\%

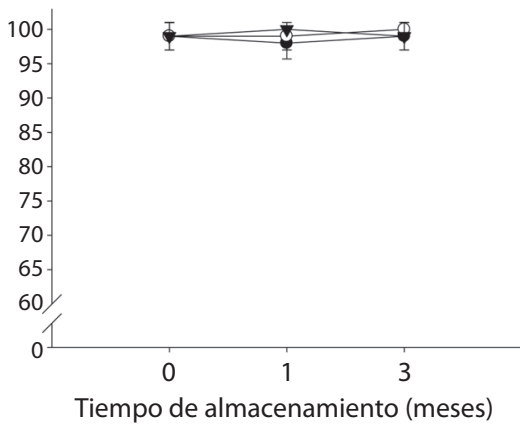

Samanea saman C.H. 6.1\%

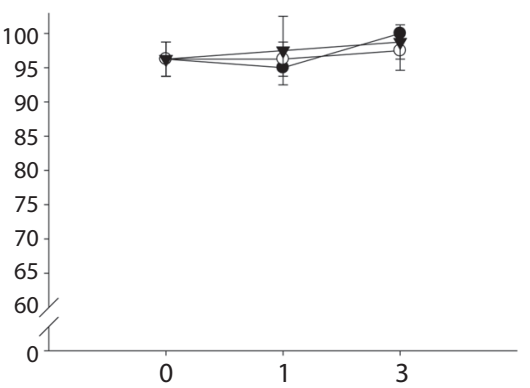

Pithecellobium dulce C.H. 5.5\%

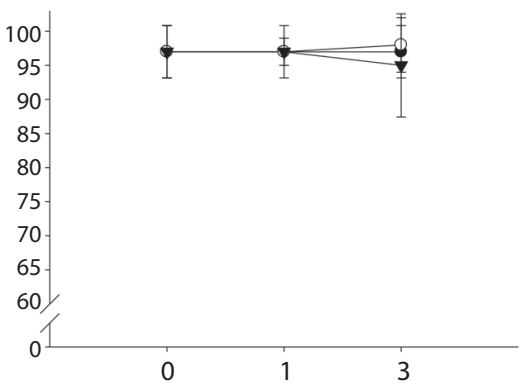

Jacaranda caucana C.H. 3.5\%

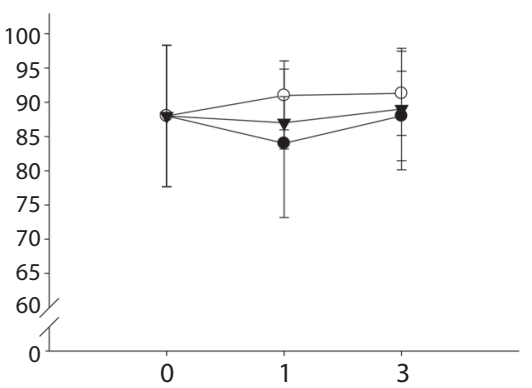

Tabebuia rosea C.H. 3.6\%

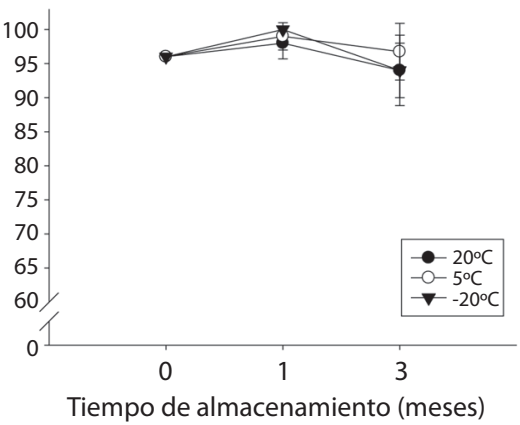

Fig. 2. Germinación de semillas de dos especies de Fabaceae y dos de Bignoniaceae, con dos condiciones de contenido de humedad, almacenadas por uno y tres meses. Las barras verticales representan el error estándar de la media.

Fig. 2. Seed germination of two Fabaceae and two Bignoniaceae species, with two moisture contents, stored for one and three months. Vertical bars represent the standard error of the mean. 
con la temperatura $20 / 30^{\circ} \mathrm{C}$ con fotoperiodo de 12 horas de luz como el tratamiento óptimo, en comparación con una germinación alta y uniforme en $P$. dulce. Esto puede indicar que las condiciones ambientales con mayor luminosidad favorecen la germinación de S. saman, lo que le puede conferir a esta especie cierta capacidad para germinar en sitios, más apropiados con el fin de que las plántulas se establezcan exitosamente. De hecho, es una especie que se establece fácilmente en sitios abiertos y altamente perturbados como los potreros activos (Esquivel et al., 2009), y es considerada potencialmente invasora en islas como las de Micronesia (Space \& Falanruw, 1999; Space, Waterhouse, Denslow, Nelson, \& Mazawa, 2000; Space et al., 2009), Samoa Americana (Space \& Flynn, 2000), Islas Cook (Space \& Flynn, 2002) y de Tonga (Space \& Flynn, 2001), por su fácil establecimiento y rápido crecimiento en sitios perturbados. Sin embargo, el hecho de que las semillas de $S$. saman tiendan a germinar menos en la oscuridad, y que la mayoría de las semillas no germinadas mueran, independientemente de la temperatura de incubación en la cual se encuentran, indica que efectivamente una parte de la población de semillas colectadas de S. saman requiere de luz para germinar. En contraste, el éxito germinativo de las semillas de $P$. dulce en todas las condiciones probadas, sugiere una capacidad potencial para colonizar sitios con diferentes calidades lumínicas. Esta especie se ha reportado como potencialmente invasora en sitios como Vietnam (Tan et al., 2012), la isla de Puerto Rico en el Caribe (Kairo, Ali, Cheesman, Haysom, \& Murphy, 2003), y las islas de Micronesia (Space et al., 2000; Space et al., 2009), lo que podría relacionarse con la respuesta positiva en la germinación en todos los tratamientos de luz evaluados.

En cuanto a las especies de Bignoniaceae, la germinación en J. caucana fue relativamente similar en los tratamientos evaluados, con excepción de los realizados a la temperatura $25 / 30^{\circ} \mathrm{C}$, los cuales fueron los más bajos. En otra especie de este género, se han encontrado diferencias en la germinación en distintas condiciones de luz y temperatura (Socolowski \& Takaki, 2004). Sin embargo, en J. caucana, los valores de PG fueron menores que los obtenidos en $T$. rosea. Esta diferencia, al igual que en las especies de Fabaceae, da cuenta de un mayor potencial de colonización de $T$. rosea en sitios con diferentes calidades lumínicas y de temperatura, en comparación con J. caucana. De hecho, se ha registrado que $T$. rosea es capaz de germinar en un rango amplio de temperaturas constantes y en presencia o ausencia de luz, lo que supone un alto reclutamiento de plántulas tanto a pleno sol como en sombra (Socolowski \& Takaki, 2007), aunque puede crecer óptimamente cuando se eliminan los competidores (Mora, Valdez, Ángeles, Musálem, \& Vaquera, 2006). Este comportamiento germinativo se ha registrado en otras especies del género Tabebuia (Cabral, Barbosa, \& Simabukuro, 2003; Santos, Sugahara, \& Takaki, 2005; Stockman, Brancalion, Novembre, \& Chamma, 2007).

Debido a la respuesta positiva en la germinación de las cuatro especies en ambientes carentes de luz (en mayor o menor intensidad), estas semillas pueden presentar moléculas de fitocromos. Incluso, con base en la respuesta positiva obtenida en los tratamientos en oscuridad, se podría pensar que el fitocromo B (PhyB) está presente en estas semillas, ya que se ha demostrado que semillas de A. thaliana, que tienen deficiencia en la producción de PhyB, no germinan en oscuridad (Shinomura, Nagatani, Chory, \& Furuya, 1994). Además, se podría pensar que las semillas de las especies evaluadas presentan también fitocromo $\mathrm{D}$ (PhyD), ya que todas registraron germinación en el tratamiento con mayor temperatura promedio $\left(25 / 30^{\circ} \mathrm{C}\right)$. El PhyD es el encargado de activar la germinación a altas temperaturas (Heschel et al., 2008), con la disminución del nivel de ácido abscísico (Seo et al., 2006). Sin embargo, se considera que es muy difícil establecer con exactitud una función específica a un tipo de fitocromo en especies donde no se han caracterizado completamente las familias de genes de estas proteínas (Shinomura, 1997). 
Las semillas de $S$. saman y $P$. dulce germinan más rápido que las de $J$. caucana y $T$. rosea, después de romper la latencia física en las dos primeras. Esto puede indicar un cierto orden de emergencia en la sucesión temprana de los bosques secos tropicales, a nivel de la germinación. Con lo anterior se esperaría en un banco de plántulas, la emergencia de las dos especies de Fabaceae en primera instancia, y luego de las dos especies de Bignoniaceae. Sin embargo, se afirma que en los bosques secos, las especies con semillas aladas tienden a colonizar más rápidamente sitios degradados o fragmentados que las semillas dispersadas por vertebrados (Vieira \& Scariot, 2006), por lo que es probable que ésta dinámica de colonización dependa de las características de crecimiento y desarrollo de las plántulas de cada especie, y no de su respuesta germinativa en esos sitios. Por otro lado, de las cuatro especies estudiadas, sólo las semillas de $S$. saman podrían tener cierto grado de exigencia en las condiciones lumínicas y de temperatura para una óptima germinación, por lo que esta especie se podría priorizar en sitios con alta iluminación, mientras que $P$. dulce, $J$. caucana y $T$. rosea pueden germinar con diferentes condiciones lumínicas.

El comportamiento ortodoxo en el almacenamiento encontrado en las semillas de $S$. saman y $P$. dulce, se ha registrado en varias especies de Fabaceae (Jayasuriya, Wijetunga, Baskin, \& Baskin, 2013). De acuerdo con Thompson (2000), se puede predecir que estas semillas con testa dura, tienen una longevidad alta, tanto en condiciones ex situ, como en condiciones in situ de bancos de semillas. De manera similar, J. caucana y $T$. rosea tienen semillas ortodoxas, las cuales son liberadas del fruto con contenidos de humedad muy bajos. Este comportamiento en el almacenamiento se ha observado en otras especies de Bignoniaceae (Degan, Aguiar, Sader, Perecin, \& Pinto, 2001; Cabral et al., 2003; Scalon, Mussury, Filho, Francelino, \& Florencio, 2006; Carvalho, Da Silva, \& Davide, 2006; Vargas-Figueroa, 2012). El almacenamiento de semillas con una alta deshidratación, incrementa el período medio de viabilidad (Murdoch \& Ellis, 2000), lo que sugiere que las semillas de estas especies podrían persistir en bancos de semillas, como lo hacen exitosamente en condiciones ex situ. Incluso, se ha registrado una tolerancia a la desecación ambiental en semillas germinadas de Tabebuia impetiginosa (Vieira, Da Silva, Alvarenga, Castro, \& Toorop, 2010), por lo que es posible que las semillas de las especies evaluadas sean tolerantes a la deshidratación en las condiciones ambientales de los bosques secos tropicales, ya que hay una mayor cantidad de especies con semillas secas y tolerantes a la deshidratación en estos ecosistemas (Vieira $\&$ Scariot, 2006). No, obstante, se ha registrado una cierta pérdida de la viabilidad en las semillas de Jacaranda copaia en experimentos de enterramiento (Dalling, Swaine, \& Garwood, 1997), por lo que dicha correspondencia de la viabilidad entre condiciones ex situ e in situ puede variar de una especie a otra.

Se concluye que las semillas de P. dulce, $J$. caucana y $T$. rosea presentan una germinación alta a diferentes calidades de luz y fluctuaciones de temperatura, por lo que es probable que germinen satisfactoriamente en sitios naturales con características diversas, como pastizales, rastrojos, bosques perturbados o bosques secundarios en estadios tempranos de sucesión vegetal, donde las condiciones microclimáticas pueden variar mucho temporal y espacialmente (Holl, 1999; Pezzopane, Reis, Reis, Da Costa, \& Chavez, 2002). Por lo tanto, se sugiere utilizar semillas de estas tres especies en procesos de restauración ecológica debido a que toleran y germinan en condiciones ambientales con amplios intervalos de temperatura y luminosidad. Por el contrario, las semillas de $S$. saman presentan una tendencia a germinar mejor en condiciones de alta luminosidad, por lo cual se sugiere utilizarla en sitios abiertos como claros de bosques y eventualmente en sitios menos luminosos, ya que pueden germinar en condiciones con relación baja de R/RL, como la encontrada en sotobosques (Pons, 2000). Debido a que los requerimientos de las semillas y las plántulas de una misma especie pueden ser diferenciales (Socolowski, Viera, \& Takaki, 2008), para determinar la capacidad de establecimiento 
y colonización de estas especies, es importante realizar estudios con plántulas, donde se pueda evaluar su crecimiento y desarrollo en distintas condiciones de luminosidad y temperatura, para así establecer los factores limitantes para estas especies en esta etapa del ciclo de vida, crucial para una permanencia de los individuos a largo plazo (Smith, 2000). Por otro lado, las semillas de las cuatro especies evaluadas pueden ser almacenadas con contenidos de humedad bajos y temperaturas bajas, sin detrimento de su viabilidad, para ser utilizadas en programas de regeneración y restauración ecológica. Además, son especies potencialmente formadoras de bancos de semillas, por su comportamiento ortodoxo. Determinar la longevidad de las semillas en condiciones naturales de los bancos es el próximo paso para estimar el tiempo que éstas pueden permanecer viables, es decir, evaluar su persistencia en bancos de semillas en condiciones naturales.

\section{AGRADECIMIENTOS}

A Katherine Lorena Rivera y María Paula Moreno, integrantes del Grupo de Investigación "Ecología y Diversidad Vegetal" de la Universidad del Valle, por su importante ayuda durante el registro de las pruebas de laboratorio. A Philip A. Silverstone Sopkin por su ayuda en la traducción del resumen. Y a la Vicerrectoría de Investigaciones de la Universidad del Valle por la financiación del presente trabajo.

\section{RESUMEN}

Las estrategias de restauración ecológica en ecosistemas altamente amenazados como el bosque seco tropical, dependen del conocimiento de factores limitantes de procesos biológicos, como la germinación y la longevidad de semillas de especies típicas de los mismos. En este estudio se evaluó el efecto de la luz y la temperatura en la germinación de semillas de dos especies de Fabaceae y dos de Bignoniaceae potencialmente útiles para restauración, y se determinó su comportamiento en el almacenamiento. Para el primer objetivo, se utilizaron cuatro niveles de calidad lumínica (fotoperiodo con 12 horas de luz, oscuridad y estímulos de luz enriquecida en rojo y luz enriquecida en rojo lejano, ambas por una hora), combinados con tres niveles de temperaturas alternadas $(20 / 25,20 / 30$ y
$25 / 30^{\circ} \mathrm{C}-16 / 8 \mathrm{~h}$ ). Para el segundo objetivo, se utilizaron dos niveles de contenido de humedad de las semillas, con tres niveles de temperatura de almacenamiento (20, 5 y $-20^{\circ} \mathrm{C}$ ) y dos tiempos de almacenamiento (1 y 3 meses). La variable de respuesta para ambos experimentos fue el número de semillas germinadas, expresado en porcentaje (PG). Samanea saman y Jacaranda caucana presentaron un PG significativamente diferente entre los tratamientos lumínicos, con el valor menor en la oscuridad, mientras Pithecellobium dulce y Tabebuia rosea no presentaron diferencias significativas entre tratamientos ( $\mathrm{PG}>90 \%$ ). La respuesta a la temperatura mostró que $25 / 30^{\circ} \mathrm{C}$ es el régimen más adecuado para promover la germinación de la mayoría de las especies. Las semillas de las cuatro especies evaluadas presentaron un comportamiento ortodoxo en el almacenamiento. Se concluye que las semillas de $P$. dulce, $J$. caucana y $T$. rosea son indiferentes a las condiciones de luz y temperatura probadas, confiriéndoles ciertas ventajas en procesos de colonización y establecimiento frente a $S$. saman, cuya germinación no se ve favorecida en la oscuridad. Se sugiere el uso de semillas de P. dulce, J. caucana y T. rosea en proyectos de restauración ecológica del bosque seco Tropical, debido a su tolerancia y germinación en condiciones ambientales con intervalos amplios de temperatura y luminosidad. Mientras que, semillas de $S$. saman podrían ser usadas en sitios abiertos como claros de bosques.

Palabras clave: Samanea saman, Pithecellobium dulce, Jacaranda caucana, Tabebuia rosea, respuesta a la luz, comportamiento de las semillas en el almacenamiento.

\section{REFERENCIAS}

Benvenuti, S., Macchia, M., \& Miele, S. (2001). Light, temperature and burial depth effects on Rumex obtusifolius seed germination and emergence. Weed Research, 41, 177-186.

Bradshaw, A. D. (1987). Restoration: an acid test for ecology. In W. R. Jordan III, M. E. Gilpin, \& J. D. Aber (Eds.), Restoration ecology, a synthetic approach to ecological research (pp. 23-29). Cambridge, United Kingdom: Cambridge University Press.

Cabral, E. L., Barbosa, D. C. A., \& Simabukuro, E. A. (2003). Armazenamento e germinação de sementes de Tabebuia aurea (Manso) Benth. \& Hook. f. ex S. Moore. Acta Botanica Brasilica, 17(4), 609-617.

Carvalho, L. R., Da Silva, E. A. A., \& Davide, A. C. (2006). Classificação de sementes florestais quanto ao comportamento no armazenamento. Revista Brasileira de Sementes, 28(2), 15-25.

Corporación Autónoma Regional del Valle del Cauca. (1990). Comparación de coberturas de bosques y humedales entre 1957 y 1986 con delimitación de las comunidades naturales críticas en el valle geográfico del río Cauca (Informe No. 90-7). Cali, Colombia. 
Dalling, J. W., Swaine, M. D., \& Garwood, N. C. (1997). Soil seed bank community dynamics in seasonally moist lowland tropical forest, Panama. Journal of Tropical Ecology, 13(5), 659-680.

Degan, P., Aguiar, I. B., Sader, R., Perecin, D., \& Pinto, L. R. (2001). Influência de métodos de secagem na conservação de sementes de Ipê-branco. Revista Brasileira de Engenharia Agrícola e Ambiental, 5(3), 492-496.

Díaz-Martín, R. M. (2005). Sinopsis de las especies colombianas de Pithecellobium (Leguminosae: Mimosoideae: Ingeae). In E. Forero, \& C. Romero (Eds.), Estudios en leguminosas colombianas (pp. 281-300). Bogotá, D.C.: Academia Colombiana de Ciencias Exactas, Físicas y Naturales, Colección Jorge Álvarez Llenas No. 25.

Ellis, R. H., Hong, T. D., \& Roberts, E. H. (1991). An intermediate category of seed storage behaviour? II. Effects of provenance, immaturity and imbibition on desiccation-tolerance in coffee. Journal of Experimental Botany, 42(238), 653-657.

Escobar, D. F., \& Torres, A. M. (2013). Morphology, ecophysiology and germination of seeds of the neotropical tree Alibertia patinoi (Rubiaceae). Revista de Biología Tropical, 61(2), 547-556.

Espinal, S., \& Montenegro, E. (1963). Formaciones vegetales de Colombia: memoria explicativa sobre el mapa ecológico. Bogotá, D.C.: Instituto Geográfico Agustin Codazzi.

Esquivel, M. J., Harvey, C. A., Finegan, B., Casanoves, F., Skarpe, C., \& Nieuwenhuyse, A. (2009). Regeneración natural de árboles y arbustos en potreros activos de Nicaragua. Agroforestería en las Américas, 47, 76-84.

Fenner, M., \& Thompson, K. (2005). The ecology of seeds. New York: Cambridge University Press.

Gentry, A. H. (1995). Diversity and floristic composition of neotropical dry forest. In S. H. Bullock, H. A. Mooney, \& E. Medina (Eds.), Seasonally tropical dry forest (pp. 146-194). Cambridge, Reino Unido: Cambridge University Press.

Gentry, A. H. (2009). Flora de Colombia No.25: Bignoniaceae. Bogotá, D.C.: Universidad Nacional de Colombia.

Harrington, J. F. (1972). Seed storage and longevity. In T. T. Kozlowski (Ed.), Seed biology, Vol. III. Insects and seed collection, storage, testing and certification (pp. 145-245). London: Academic Press.

Heschel, M. S., Butler, C. M., Barua, D., Chiang, G., Wheeler, A., Sharrock, R. A., \& Donohue, K. (2008). New roles of phytochromes during seed germination. International Journal of Plant Sciences, 169(4), 531-540.
Holl, K. D. (1999). Factors limiting tropical rain forest regeneration in abandoned pasture: seed rain, seed germination, microclimate, and soil. Biotropica, 31(2), 229-242.

Hong, T. D., \& Ellis, R. H. (1996). A protocol to determine seed storage behaviour. (Technical Bulletin No. 1) Retrieved from International Plant Genetic Resource Institute http://www.cbd.int/doc/case-studies/tttc/ SeedStorage.pdf

Instituto de Hidrología, Meteorología y Estudios Ambientales. (2005). Atlas climatológico de Colombia. Retrieved from http://institucional.ideam.gov.co/jsp/ loader.jsf?1Servicio=Publicaciones \&lTipo=publicaci ones\&lFuncion=loadContenidoPublicacion $\& \mathrm{id}=55$

International Seed Testing Association. (1999). International Rules for Seed Testing. Zürich, Switzerland: Seed Science \& Technology.

Janzen, D. H. (1988). Tropical dry forests, the most endangered major tropical ecosystem. In E. O. Wilson, \& F. M. Peter (Eds.), Biodiversity (pp. 130-144). Washington, DC: National Academies Press.

Jayasuriya, K. M. G. G., Wijetunga, A. S. T. B., Baskin, J. M., \& Baskin, C. C. (2013). Seed dormancy and storage behavior in tropical Fabaceae: a study of 100 species from Sri Lanka. Seed Science Research, 23(4), 257-269.

Kairo, M., Ali, B, Cheesman, O., Haysom, K., \& Murphy, S. (2003). Invasive species threats in the Caribbean region (Report to The Nature Conservancy). Retrieved from http://www.issg.org/database/species/reference files/Kairo\%20et\%20al,\%202003.pdf

Kendrick, R. E., \& Spruit, C. J. P. (1977). Phototransformation of phytochrome. Photochemistry and Photobiology, 26, 201-214.

Labouriau, L. G., \& Valadares, M. E. (1976). On the germination of seeds of Calotropis procera (Ait.) Ait.f. Anais da Academia Brasileira de Ciências, 48(2), 263-284.

McCullough, J. M., \& Shropshire, W. J. (1970). Physiological predetermination of germination responses in Arabidopsis thaliana (L.) Heynh. Plant \& Cell Physiology, 11, 139-148.

Mora, A., Valdez, J. I., Ángeles, G., Musálem, M. A., \& Vaquera, H. (2006). Establecimiento y desarrollo de plántulas de Tabebuia rosea (Bignoniaceae) en una selva subcaducifolia manejada de la costa Pacífica de México. Revista de Biología Tropical, 54(4), 1215-1225.

Murphy, P. G., \& Lugo, A. E. (1995). Dry forests of Central America and the Caribbean. In S. H. Bullock, H. A. Mooney, \& E. Medina (Eds.), Seasonally tropical dry forest (pp. 9-34). Cambridge, United Kingdom: Cambridge University Press. 
Murdoch, A. J., \& Ellis, R. H. (2000). Dormancy, viability and longevity. In M. Fenner, (Ed.), Seeds: the ecology of regeneration in plant communities (pp. 183-214). London: $\mathrm{CAB}$ International.

Nichols, M. A., \& Heydecker, W. (1968). Two approaches to the study of germination data. Proceedings of the International Seed Testing Association, 33(3), 531-540.

Pérez-Arbeláez, E. (1956). Plantas útiles de Colombia. Bogotá, D.C.: Camacho Roldán Ltda.

Pezzopane, J. E. M., Reis, G. G., Reis, M. G. F., Da Costa, J. M. N., \& Chaves, J. H. (2002). Temperatura do solo no interior de um fragment de floresta secundária semidecidual. Revista Brasileira de Agrometeorologia, 10(1), 1-8.

Pons, T. L. (2000). Seeds responses to light. In M. Fenner (Ed.), Seeds: the ecology of regeneration in plant communities (pp. 237-260). London: CAB International.

Portillo-Quintero, C. A., \& Sánchez-Azofeifa, G. A. (2010). Extent and conservation of tropical dry forest in the Americas. Biological Conservation, 143, 144-155.

Primack, R. B. (1980). Variation in the phenology of natural population of montane shrubs in New Zealand. Journal of Ecology, 68(3), 849-862.

Probert, R. J. (2000). The role of temperature in the regulation of seed dormancy and germination. In M. Fenner (Ed.), Seeds: the ecology of regeneration in plant communities (pp. 261-292). London: CAB International.

Quesada, M., Sánchez-Azofeifa, A., Álvarez-Añorve, M., Stoner, K. E., Avila-Cabadilla, L., Calvo-Alvarado, J., \& Sánchez-Montoya, G. (2009). Succession and management of tropical dry forests in the Americas: Review and new perspectives. Forest Ecology and Management, 258, 1014-1024.

Ranal, M. A., \& Santana, D. G. (2006). How and why to measure the germination process? Revista Brasileira de Botanica, 29(1), 1-11.

Roberts, E. H. (1973). Predicting the storage life of seed. Seed Science \& Technology, 1, 499-514.

Santos, D. L. D., Sugahara, V. Y., \& Takaki, M. (2005). Efeitos da luz e da temperatura na germinação de sementes de Tabebuia serratifolia (Vahl) Nich, Tabebuia chrysotricha (Mart. ex DC.) Standl. e Tabebuia roseo-alba (Ridl) Sand - Bignoniaceae. Ciência Florestal, 15(1), 87-92.

Scalon, S. P. Q., Mussury, R. M., Filho, H. S., Francelino, C. S. F., \& Florencio, D. K. A. (2006). Armazenamento e tratamentos pré-germinativos em sementes de jacarandá (Jacaranda cuspidifolia Mart.). Revista Árvore, 30(2), 179-185.

Seo, M., Hanada, A., Kuwahara, A., Endo, A., Okamoto, M., Yamauchi, Y., \& Nambara, E. (2006). Regulation of hormone metabolism in Arabidopsis seeds: phytochrome regulation of abscisic acid metabolism and abscisic acid regulation of gibberellin metabolism. The Plant Journal, 48, 354-366.

Shinomura, T. (1997). Phytochrome regulation of seed germination. Journal of Plant Research, 110, 151-161.

Shinomura, T., Nagatani, A., Chory, J., \& Furuya, M. (1994). The induction of seed germination in Arabidopsis thaliana is regulated principally by phytochrome B and secondarily by phytochrome A. Plant Physiology, 104(2), 363-371.

Siegel, S., \& Castellan, N. J. (1988). Nonparametric statistics for the behavioral sciences. New York: McGraw-Hill.

Smith, H. (2000). Phytochromes and light signal perception by plants - an emerging synthesis. Nature, 407, 585-591.

Socolowski, F., \& Takaki, M. (2004). Germination of Jacaranda mimosifolia (D. Don - Bignoniaceae) seeds: effects of light, temperature and water stress. Brazilian Archives of Biology and Technology, 47, 785-792.

Socolowski, F., \& Takaki, M. (2007). Germinação de sementes e emergência de plântulas de Tabebuia rosea (Bertoloni) A.P. De Candolle (Bignoniaceae), uma espécie exótica com potencial invasor. Revista Árvore, 31(2), 229-238.

Socolowski, F., Vieira, D. C. M., \& Takaki, M. (2008). Interaction of temperature and light on seed germination in Tecoma stans L. Juss. ex Kunth (Bignoniaceae). Brazilian Archives of Biology and Technology, 51, 723-730.

Space, J. C., \& Falanruw, M. (1999). Observations on invasive plant species in Micronesia. (Report prepared for the meeting of the Pacific Islands Committee, Council of Western State Foresters, Majuro, Republic of the Marshall Islands). Retrieved from http://www. hear.org/pier/pdf/micronesia_report.pdf

Space, J. C., \& Flynn, T. (2000). Observations on invasive plant species in American Samoa (Report prepared for the meeting of the Pacific Islands Committee, Council of Western State Foresters, Majuro, Republic of the Marshall Islands). Retrieved from http://www. hear.org/pier/pdf/american_samoa_report.pdf

Space, J. C., \& Flynn, T. (2001). Report to the Kingdom of Tonga on invasive plant species of environmental concern. Retrieved from Institute of Pacific Islands Forestry http://www.hear.org/pier/pdf/tonga_report. pdf

Space, J. C., \& Flynn, T. (2002). Report to the government of the Cook Islands in invasive plant species of environmental concern. Retrieved from Institute of Pacific Islands Forestry http://www.hear.org/Pier/pdf/ cook_islands_report.pdf 
Space, J. C., Lorence, D. H., \& LaRosa, A. M. (2009). Report to the Republic of Palau: 2008 update on invasive plant species. Retrieved from Institute of Pacific Islands Forestry http://hear.its.hawaii.edu/ pier/pdf/Palau_report_2008.pdf

Space, J. C., Waterhouse, B., Denslow, J. S., Nelson, D., \& Waguk, E. E. (2000). Invasive plant species on Kosrae, Federated States of Micronesia. Retrieved from Institute of Pacific Islands Forestry http://www.hear. org/pier/pdf/kosrae_report.pdf

Stockman, A. L., Brancalion, P. H. S., Novembre, A. D. L. C., \& Chamma, H. M. C. P. (2007). Sementes de Ipê-Branco (Tabebuia roseo-alba (Ridl.) Sand. - Bignoniaceae): temperatura e substrato para o teste de germinação. Revista Brasileira de Sementes, 29(3), 139-143.

Tan, D. T., Thu, P. Q., \& Dell, B. (2012). Invasive plant species in the national parks of Vietnam. Forests, 3, 997-1016.

Thompson, K. (2000). The functional ecology of soil seed banks. In M. Fenner (Ed.), Seeds: the ecology of regeneration in plant communities (pp. 215-235). London: CAB International.

Torres, A. M., Adarve, J. B., Cárdenas, M., Vargas, J. A., Londoño, V., Rivera, K., \& González, A. M. (2012). Dinámica sucesional de un fragmento de bosque seco tropical del Valle del Cauca, Colombia. Biota Colombiana, 13(2), 66-85.

Vargas-Figueroa, J. A. (2012). Fisiología de semillas de Tecoma stans (Bignoniaceae), una especie del bosque seco tropical. (Unpublished bachelor thesis). Universidad del Valle, Cali, Colombia.

Vieira, C. V., Da Silva, E. A. A., Alvarenga, A. A., Castro, E. M., \& Toorop, P. E. (2010). Stress-associated factors increase after desiccation of germinated seeds of Tabebuia impetiginosa Mart. Plant Growth Regulation, 62, 257-263.

Vieira, D. L. M., \& Scariot, A. (2006). Principles of natural regeneration of tropical dry forests for restoration. Restoration Ecology, 14(1), 11-20.

Zar, J. H. (1996). Biostatistical analysis (3 ${ }^{\text {rd }}$ edition). Upper Saddle River, New Jersey: Prentice Hall. 
